\title{
Uso de un ambiente virtual de aprendizaje en la ingeniería en sistemas computacionales del Tecnológico de Ciudad Valles como herramienta de apoyo a las clases presenciales
}

\section{Use of a virtual learning environment in computer systems engineering at the Tecnológico de Ciudad Valles as a support tool for face-to-face classes}

GARCIA-CHI, Rosa Imelda*†, HERNANDEZ, María Antonieta, IZAGUIRRE-CÁRDENAS, Nelly Rosina y EGUIA-ALVAREZ, Arturo

SEP, Tecnológico Nacional de México. Instituto Tecnológico de Ciudad Valles, Carretera al Ingenio Km 2. Col. Vista Hermosa, CP. 79010, Cd. Valles, SLP, México.

ID $1^{\text {er }}$ Autor: Rosa Imelda, García-Chi / ORC ID: 0000-0001-8462-2859, Research ID: RosyChi 79010-4811227776, arXiv ID Author: RosyChi, CVU CONACYT ID: 998355

ID $1^{\text {er }}$ Coautor: María Antonieta, Hernández / ORC ID 0000-0002-5568-3924, Research ID: antonieta 79030-4813840779, arXiv ID Author: Antonieta, CVU CONACYT ID: 998431

ID $2^{\text {do }}$ Coautor: Nelly Rosina, Izaguirre-Cárdenas / ORC ID 0000-0003-4707-2389, Research ID: NellyIzaguirre 790104811286386, arXiv ID Author:NellyIzaguirre, CVU CONACYT ID: 997704

ID $3^{\text {er }}$ Coautor: Arturo, Eguía-Alvarez, ORC ID: 0000-0003-3034-3066, CVU CONACYT ID: 997835

DOI: $10.35429 / J A D \cdot 2019.8 .3 .7 .14$

Recibido 15 de Abril, 2019; Aceptado 22 de Junio, 2019

\section{Resumen}

El artículo es el resultado de un proyecto de investigación educativa del Tecnológico Nacional de México que involucra Ambientes Virtuales de Aprendizaje (AVA). El objetivo es determinar el impacto de usar un AVA como herramienta en el proceso de enseñanza aprendizaje de las clases presenciales del Tecnológico de Ciudad Valles. La metodología es mixta, cualitativa y cuantitativa. En la cuantitativa se determina el número de profesores que utiliza AVA, número de alumnos en un curso Blending Learning (B-Learning), número de cursos diseñados en Moodle. En la cualitativa, se analiza el impacto del aprendizaje B-Learning en los estudiantes; determinando el grado de motivación y desempeño que se genera al usar AVA y la habilidad de utilizar la tecnología de información para generar sus estrategias de aprendizaje. Se presenta un análisis componencial y estructural del AVA utilizado en el instituto, se da a conocer la hipótesis de la investigación: el uso de un AVA como estrategia b-learning, mejora el nivel de desempeño de competencias del estudiante en comparación con estudiantes que siguen estrategias de aprendizaje presencial. Se describe la manera en que se realiza la investigación, el marco de referencia y se muestran resultados relevantes y conclusiones.

\begin{abstract}
The article is the result of an educational research project of the National Technological Institute of Mexico that involves Virtual Learning Environments (AVA). The objective is to determine the impact of using a VPA as a tool in the teachinglearning process of the face-to-face classes of the Ciudad Valles Technology. The methodology is mixed, qualitative and quantitative. The quantitative number determines the number of teachers used by AVA, the number of students in a Blending Learning (B-Learning) course, the number of courses designed in Moodle. In the qualitative, the impact of B-Learning on students is analyzed; determining the degree of motivation and performance that is generated when using AVA and the ability to use information technology to generate their learning strategies. A composite and structural analysis of the AVA used in the institute is presented, the research hypothesis is disclosed: the use of an AVA as a b-learning strategy, improves the level of performance of student competencies compared to students who follow classroom learning strategies. The manner in which the research is conducted, the frame of reference is described and relevant results and conclusions are shown.
\end{abstract}

Citación: GARCIA-CHI, Rosa Imelda, HERNANDEZ, María Antonieta, IZAGUIRRE-CÁRDENAS, Nelly Rosina y EGUIA-ALVAREZ, Arturo. Uso de un ambiente virtual de aprendizaje en la ingeniería en sistemas computacionales del Tecnológico de Ciudad Valles como herramienta de apoyo a las clases presenciales. Revista de Arquitectura y Diseño. 2019. 3-8: 7-14.

*Correspondencia al Autor (Correo Electrónico: rosa.garcia@tecvalles.mx)

$\dagger$ Investigador contribuyendo como primer autor. 


\section{Introducción}

El proyecto de investigación educativa analiza el impacto que tiene un Ambiente Virtual de Aprendizaje (AVA), como lo es el Moodle, en una modalidad B-learning, para determinar el desempeño de las competencias académicas del estudiante y su motivación al aprender versus con estudiantes que usan la modalidad presencial. El objeto de estudio son los estudiantes de la ingeniería en Sistemas Computacionales del Tecnológico Nacional de México campus Instituto Tecnológico de Ciudad Valles de la asignatura de simulación. Se utilizó una metodología mixta de investigación, es decir, cuantitativa y cualitativa. La cuantitativa se aplicó a números de: cursos, alumnos, objetos de aprendizaje y criterios de evaluación. La cualitativa al desempeño de las competencias y a la motivación del estudiante.

En el Tecnológico de Ciudad Valles la modalidad educativa es presencial, para esta investigación se adopta una modalidad Blearning para enseñar con un AVA. Utilizar el AVA, en este caso, Moodle, promueve la inclusión a la vida social, cultural, económica y laboral del siglo XXI, en la cual la tecnología tiene un lugar fundamental. En este sentido, enseñar en una modalidad B-learning usando Moodle, ayuda a los estudiantes adquirir distintas capacidades y habilidades vinculadas con el entorno de aprendizaje y la tecnología. (García-Ruiz,2018). La hipótesis alterna o de investigación, determina que el uso de un Ambiente Virtual de Aprendizaje Moodle con objetos de aprendizaje o evaluación de un curso, mejora el nivel de desempeño de competencias del estudiante, en comparación con estudiantes que son evaluados con estrategias de aprendizaje presencial.

En este artículo se da a conocer el planteamiento general del problema, el universo, los sujetos y la muestra de estudio; se describe el método y las técnicas utilizadas para llevar a cabo la investigación; se inicia con el análisis componencial y estructural del AVA, se revisan los criterios de evaluación, los objetos de aprendizaje y las estrategias de evaluación de la modalidad presencial y b-learning, se presenta información recopilada, analizada e integrada, para mostrar resultados a través de la prueba estadística de U de Mann-Whitney; se incluyen las conclusiones de la investigación.

\section{Descripción del Método}

El Tecnológico Nacional de México trabaja tres dimensiones en su modelo educativo: filosófica, académica y organizacional en su fin esencial es la formación integral del estudiante. (Gamino-Carranza, A., 2016).

\section{El Tecnológico Nacional de México} tiene un modelo educativo basado en competencias, por lo que la planeación e instrumentación de los cursos que se imparten en el Tecnológico de Ciudad Valles se diseñan bajo este modelo. (TecNM, 2012) Se utiliza un documento controlado por el Sistema de Gestión Integral para su proceso académico, denominado Instrumentación didáctica; en este documento se establecen las generalidades de la asignatura a impartir (nombre, créditos, semestre, carrera, departamento, la intención didáctica y la competencia a alcanzar), estrategias de enseñanza y estrategias de aprendizaje; establece los criterios de evaluación que miden el desempeño académico de los estudiantes.

En el modelo de competencias, se pretende alcanzar un nivel de desempeño académico, con dos opciones: Competencia alcanzada y Competencia no alcanzada. (Gutiérrez, M. A. U., 2017). La opción de desempeño de competencia alcanzada está integrada por cuatro niveles de desempeño: excelente (cumple 5 indicadores), notable (cumple 4 indicadores), bueno (cumple 3 indicadores) y suficiente (cumple 2 indicadores); mientras que la opción de desempeño de competencia no alcanzada solo tiene el nivel de desempeño insuficiente (no cumple indicadores). Estos niveles serían las variables cualitativas ordinales de la investigación. Para que el estudiante acredite una asignatura, debe ser evaluado en todas y cada una de las competencias de la misma, y el nivel de desempeño alcanzado por el estudiante estará sustentado en las evidencias y cumplimiento de los indicadores de alcance definidos en la instrumentación didáctica.

Los resultados de las evaluaciones de cada competencia se promedian para obtener la calificación de la asignatura, siempre y cuando se hayan alcanzado todas las competencias. 
Las valoraciones numéricas asignadas por el profesor, a una competencia, indican el nivel de desempeño con que el estudiante alcanzó la competencia y estará sustentada en los métodos, técnicas e instrumentos de evaluación que utilice el (la) profesor(a) para la asignatura, tomando como referencia los indicadores de alcance que se definen a continuación: a) se adapta a situaciones y sistemas complejos, b) hace aportaciones a las actividades académicas desarrolladas, c) propone y explica soluciones o procedimientos no vistos en clase, muestra creatividad, d) introduce recursos $y$ experiencias que promueven un pensamiento crítico, e) incorpora conocimientos y actividades interdisciplinarias en su aprendizaje, f) realiza su trabajo en forma autónoma y autorregulada. (TecNM, 2015)

Para este proyecto se considera como universo de investigación, los cursos creados en el Ambiente Virtual de Aprendizaje Moodle del Tecnológico de Ciudad Valles para el plan de estudios de la ingeniería en sistemas computacionales ISIC-2010-224 (OTecNM, con validez de mayo de 2016. Los sujetos de estudio son los estudiantes de la carrera de Ingeniería en Sistemas Computacionales y la muestra, los estudiantes que cursaron la asignatura de Simulación en los años 2017, 2018 y 2019.

Para llevar a cabo esta investigación se integrará una metodología mixta, tanto cualitativa como cuantitativa. Referente a la metodología cuantitativa se determinará el número de programas de estudio que se han desarrollado en el AVA, el número de alumnos que llevan un curso teniendo como herramienta de apoyo a Moodle en su proceso de aprendizaje, el número de alumnos que llevan un curso de manera presencial sin apoyo de Moodle.

En cuanto a la metodología cualitativa, se hará un análisis de los objetos de aprendizaje $\mathrm{y}$ evaluativos creados en Moodle y de las estrategias de evaluación aplicados en el aula; determinando el nivel de desempeño de la competencia que alcanza el estudiante al usar una u otra opción.
Los resultados positivos de la investigación llevarán a la construcción de programas de capacitación que promueva y fortalezca a los profesores en la creación de sus cursos en el Ambiente Virtual de Aprendizaje Moodle como apoyo al proceso de enseñanza aprendizaje presencial, para mejorar el nivel de desempeño de las competencias en los estudiantes.

\section{Metodología a desarrollar}

La metodología cuantitativa y cualitativa del proyecto, se basan en técnicas de observación, selección, verificación y análisis de información. Los instrumentos utilizados son los motores de búsqueda como Google, los lineamientos del Tecnológico Nacional de México, Plan y Programa de estudios de Ingeniería en Sistemas Computacionales TecNM () mayo 2015, Campus Virtual del Tecnológico de Ciudad Valles (Moodle), instrumentación didáctica de la asignatura de Simulación modalidad presencial y b-learning, objetos de aprendizaje y estrategias de evaluación presencial.

Los objetivos y actividades definidas para el proyecto son los siguientes:

1. Identificar el Ambiente Virtual de Aprendizaje utilizado en el Tecnológico de Ciudad Valles

a. Realizar un análisis del Ambiente Virtual de Aprendizaje Moodle sobre su estructura global y componencial

b. Identificar el modelo pedagógico de Moodle

2. Elaborar un diagnóstico referente a que programas de estudio (cursos) de la carrera de Ingeniería en Sistemas Computacionales se han desarrollado en el Moodle

a. Revisar y enlistar que cursos han sido creados en el Ambiente Virtual de Aprendizaje

b. Revisar y describir la estructura del curso creado en el AVA 
3. Identificar los objetos de aprendizaje que determinan el desempeño de evaluación de la competencia del estudiante en el Moodle

a. Revisar el diseño de la instrumentación didáctica y obtener los criterios de evaluación

b. Revisar y describir los objetos de aprendizaje que se utilizan para evaluar el estudiante en el Ambiente Virtual de Aprendizaje

c. Verificar si se ha creado los objetos de aprendizaje en relación con cada criterio de evaluación definido en la instrumentación para la consecución de la competencia de la unidad temática

4. Identificar las estrategias de evaluación que determinan el desempeño de evaluación de la competencia en el aula

a. Revisar el diseño de la instrumentación didáctica y obtener los criterios de evaluación

b. Revisar y describir las estrategias de aprendizaje que se utilizan para evaluar el estudiante de manera presencial

c. Verificar si se han creado las estrategias de aprendizaje en relación con cada criterio de evaluación definido en la instrumentación para la consecución de la competencia de la unidad temática

5. Realizar un estudio comparativo del nivel de desempeño de competencias del estudiante de Ingeniería en sistemas Computacionales del 1 IT de Cd. Valles en una asignatura determinada, comparando los que usan la plataforma Moodle versus los que no la usan.

a. Seleccionar grupo donde la asignatura cuyo curso se ha creado en Moodle y utiliza la modalidad mixta.

b. Seleccionar grupo con la misma asignatura cuyo curso sea solamente presencial c. Recopilar información sobre las calificaciones obtenidas en las unidades temáticas, el desempeño de la competencia $y$ el nivel de desempeño logrado por los estudiantes del curso que se apoyó con un AVA

d. Recopilar información sobre las calificaciones obtenidas en las unidades temáticas, el desempeño de la competencia $y$ el nivel de desempeño logrado por los estudiantes del curso que no se apoyó con un AVA

e. Realizar estudio comparativo de la recopilación de la información en cuanto al desempeño de las competencias en estudiantes con la técnica estadística de U de MannWhitney.

f. Describir el resultado del estudio elaborando un reporte del desempeño de las competencias de los estudiantes determinando el impacto en el nivel de desempeño al usar un Ambiente Virtual de Aprendizaje en el proceso de enseñanza aprendizaje

\section{Resultados}

Se describen los resultados por objetivo:

1. Se llevó a cabo el análisis Estructural y componencial de la Plataforma Moodle del Tecnológico de Cd. Valles utilizando la técnica de recopilación, observación y análisis de la información. La estructura componencial se integra por bloques: administración, configuración, calendario, perfil y rol de usuario, la columna central muestra una imagen de Bienvenida y las categorías y subcategorías que integra el Campus Virtual del Tecnológico de $\mathrm{Cd}$. Valles; este análisis corresponde a la investigación cualitativa. Ver la figura 1. 


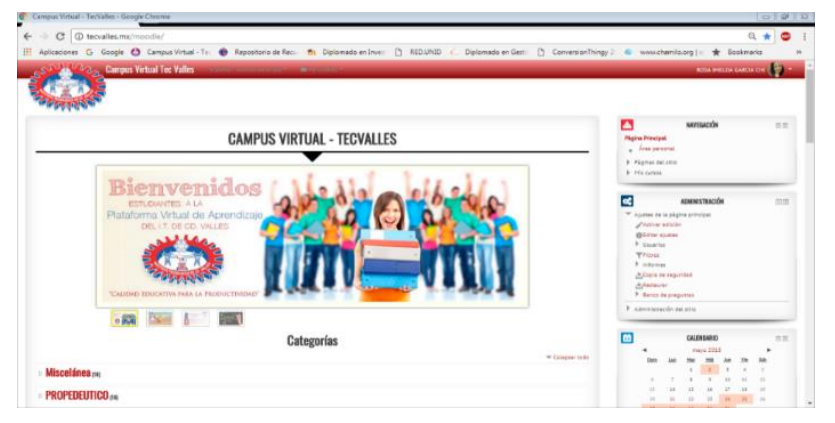

Figura 1 Plataforma Moodle

Fuente: www.tecvalles.mx

2. A través de la técnica de la observación y análisis de información, se analizan de manera cuantitativa las categorías que el Administrador de la plataforma ha creado. La diversidad de categorías que cubre, se relaciona con las carreras que ofrece el Tecnológico de Ciudad Valles. Ver Figura 2.

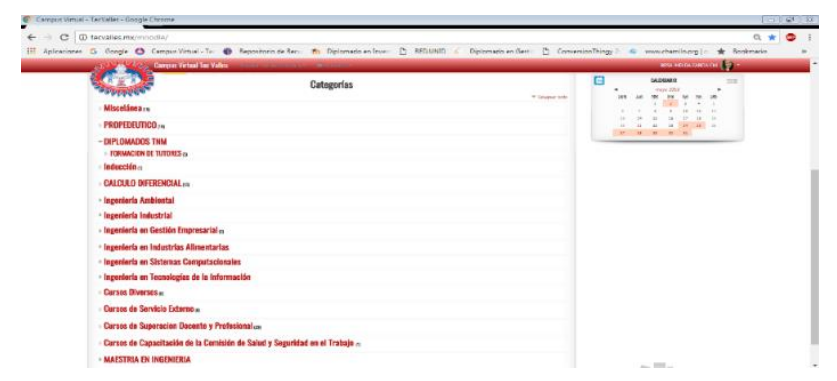

Figura 2 Categorías del Campus Virtual del Tec Valles Fuente: www.tecvalles.mx

Se selecciona la categoría delimitada para el proyecto de investigación educativa, la de Ingeniería en Sistemas Computacionales, se analizan las subcategorías que define los semestres, la especialidad y las tutorías que se ofrecen para esa carrera. Se lleva a cabo el análisis cuantitativo contabilizando los cursos y se elabora un cuadro donde se registra el semestre, el curso y el docente que ha elaborado ese curso en la plataforma Moodle del Tecnológico de Cd. Valles, se tienen 48 cursos en 11 subcategorías. Se toma para fines de la investigación el curso de Simulación que se encuentra en la subcategoría 4to semestre de Ingeniería en Sistemas Computacionales para hacer una revisión de los criterios de evaluación definidos en la instrumentación didáctica del profesor. Los grupos analizados son los de las claves ISI55A de la maestra Rosa Imelda García Chi, el grupo ISI55B de la maestra María Antonieta Hernández que usa la modalidad b-learning.
3. Para este objetivo se lleva a cabo una investigación cualitativa y cuantitativa. Se inicia con la técnica de la recopilación de la Instrumentación didáctica y luego con la técnica de la observación. A partir del análisis de los criterios de evaluación que se establecen en el instrumentación didáctica se determina cuantitativamente cuantos objetos de aprendizaje han sido creados, entre actividades de aprendizaje y recursos de enseñanza; se revisan los objetos de aprendizaje generados en el campus virtual para verificar la correspondencia con lo establecido en la instrumentación y evaluar el nivel de desempeño que el estudiante puede alcanzar y que contribuya a su proceso cognitivo de aprendizaje. Ver Figura 3.

Bienvenidos al Curso de Simulación

Protesoro: MTL. Rosa Imeida Garcia Chi

Competencia Especifica: Analizar, modelar, desarrollar y experimentar sistemas productivos y de servicios, reales o hipotéticos, a través de la simulación de eventos discretos, con el fin de conocerlos con claridad o mejorar su funcionamiento, aplicando herramientas matemáticas y de programación.

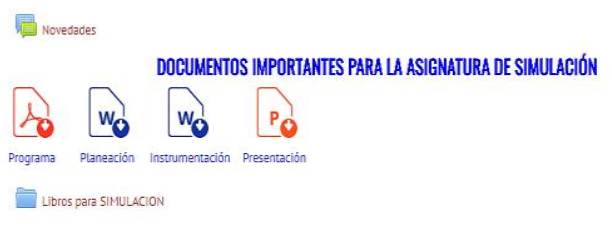

Figura 3 Curso de Simulación en Moodle Fuente:http://tecvalles.mx/moodle/course/view.php?id=255

Se elabora un registro de que objetos se desarrollan, con que herramienta y como aporta al proceso de aprendizaje del estudiante al interactuar con el objeto aportando información cualitativa a la investigación.

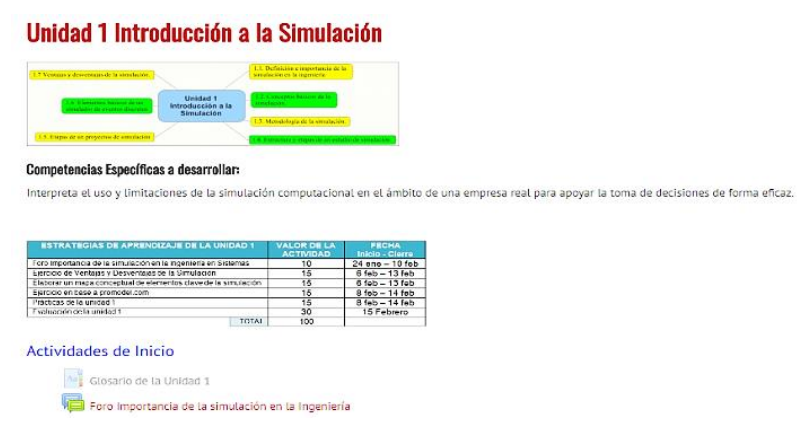

Figura 4 Unidad 1 del curso de Simulación en Moodle Fuente:http://tecvalles. $m x /$ moodle/course/view.php?id=2 55 


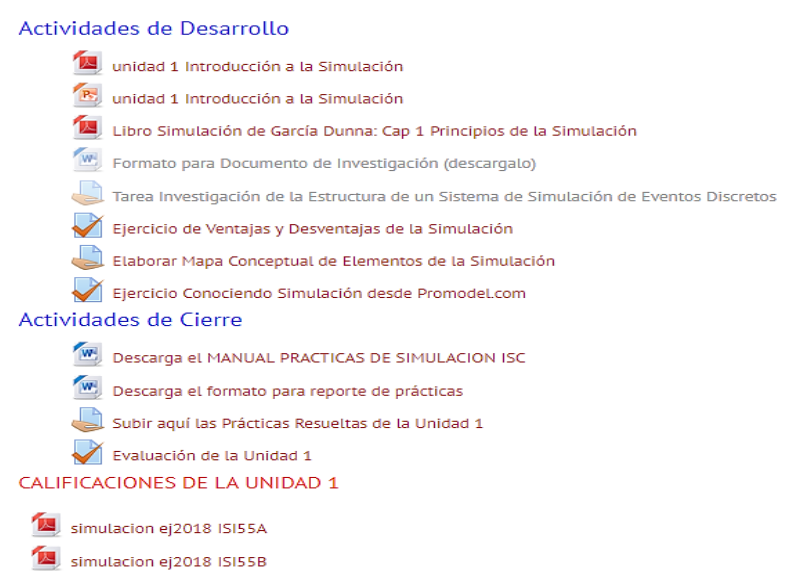

Figura 5 Actividades de la unidad 1 del curso de Simulación en Moodle

Fuente:

moodle/course/view.php?id=255

http://tecvalles.mx/

Se describe el porcentaje o puntuación que aporta el objeto de aprendizaje al nivel de desempeño según los criterios de evaluación establecidos en la instrumentación didáctica.

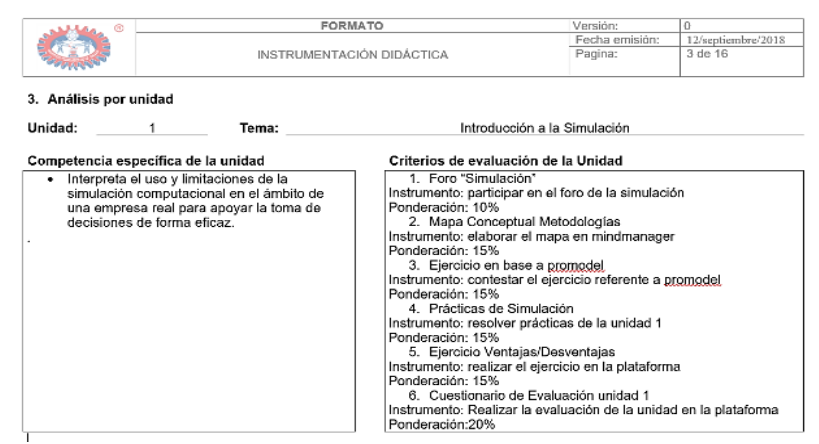

Figura 6 Instrumentación Didáctica del curso de Simulación

Fuente: documento de instrumentación didáctica

4. La técnica de la recopilación, observación y análisis de la información se utilizaron en este punto. Se recopiló la instrumentación didáctica con el maestro que imparte la materia de Simulación en la modalidad presencial, el grupo ISI55C a cargo del maestro Arturo Eguía Álvarez. Una vez obtenida, se analiza y se extraen de manera cuantitativa los criterios que utiliza para medir el desempeño del estudiante por cada unidad de aprendizaje. También se recopilan algunas de las estrategias de aprendizaje que utiliza en el aula, estas muestran las características cualitativas que debe tener un instrumento de evaluación para evaluar el aprendizaje factual.

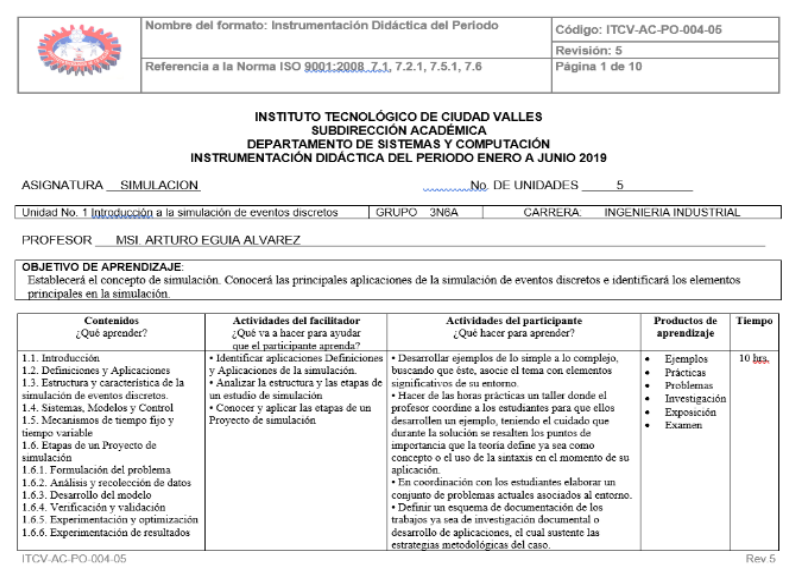

Figura 7 Instrumentación Didáctica del curso de Simulación presencial

Fuente: documento de instrumentación didáctica

5. En este objetivo se llevó a cabo la selección de los grupos a ser utilizados en la investigación, tanto los de la modalidad blearning y la modalidad presencial. Los grupos seleccionados corresponden a la materia de Simulación de la carrera de Ingeniería en sistemas Computacionales, los grupos son el ISI55A a cargo de la maestra Rosa Imelda García Chi, el grupo ISI55B a cargo de la maestra María Antonieta Hernández y el grupo ISI55C a cargo del maestro Arturo Eguía Álvarez. Se utiliza la técnica de la recopilación donde se recaban las actas de calificaciones de los tres grupos, como se muestra en la figura 8,9 y 10 .
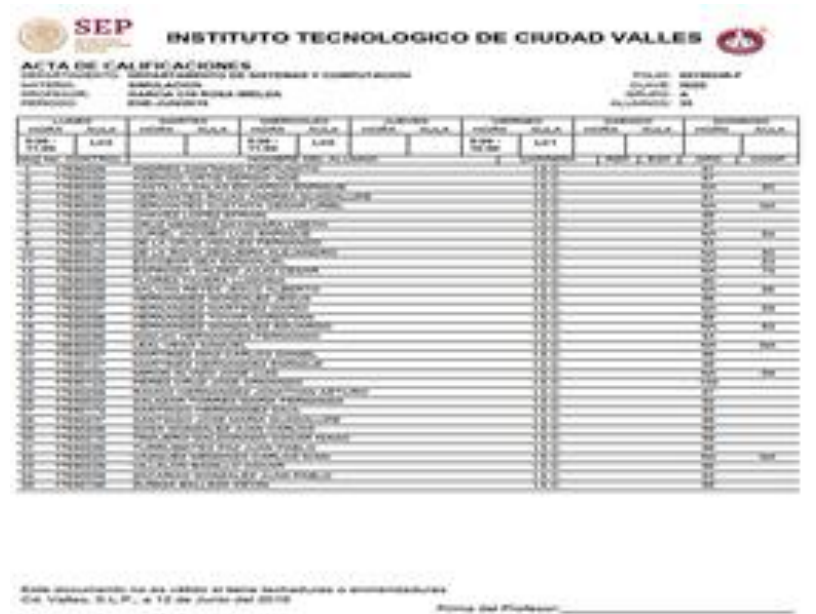

Figura 8 Acta de calificaciones grupo ISI55A

Fuente: www.tecvalles. $\mathrm{x} x / \mathrm{SII}$ 


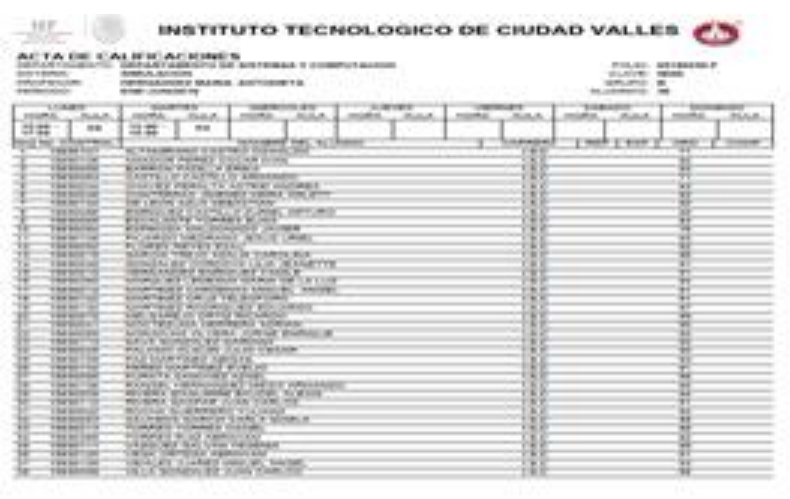

Figura 9 Acta de calificaciones grupo ISI55B Fuente: $w w w . t e c v a l l e s . m x / S I I$

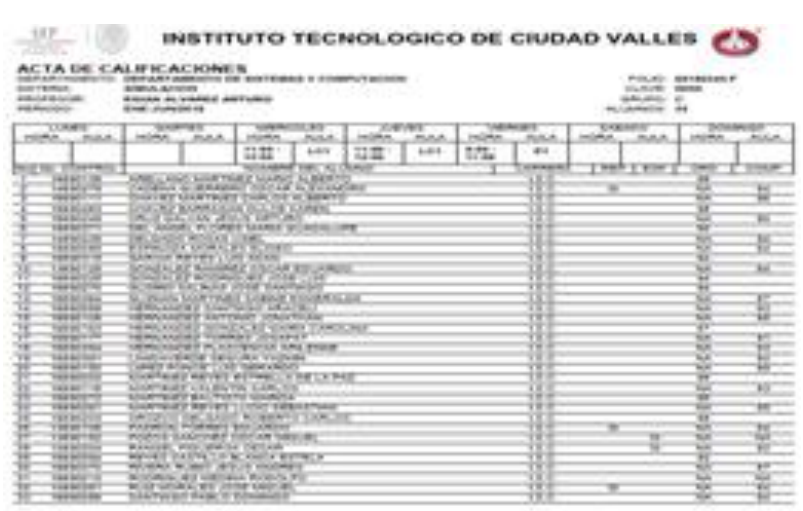

Figura 10 Acta de calificaciones grupo ISI55C Fuente: www.tecvalles.mx/SII

Para determinar el impacto del uso de un ambiente virtual de aprendizaje como apoyo a las clases presenciales, se utiliza la técnica estadística U de Mann-Whitney con el software SPSS. La variable cualitativa y las cualitativa ordinal son la modalidad educativa y el nivel de desempeño.

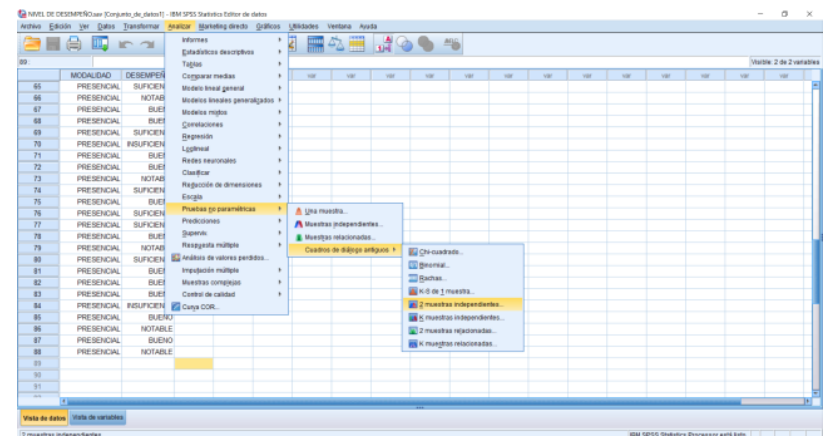

Figura 11 Prueba estadística de U de Mann-Whitney para variables cualitativas ordinales Fuente: Software IBM SPSS Stastistics
Se realiza el cálculo de pruebas no paramétricas de dos muestras independientes; se obtiene la siguiente información del software IBM SPSS:

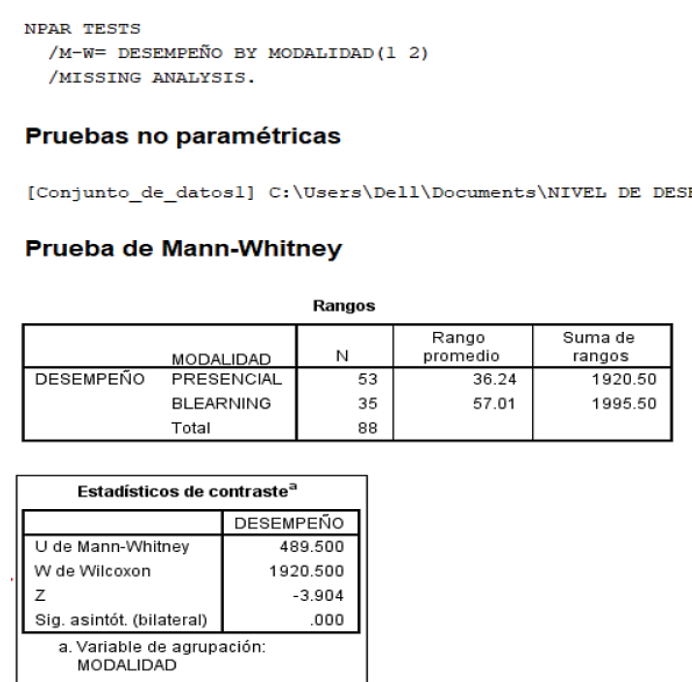

Figura 12 Resultado de la prueba estadística de U de Mann-Whitney

Fuente: Software IBM SPSS Stastistics

Si $\mathrm{z} \leq 0.05$ la hipótesis nula se rechaza y la hipótesis de investigación se acepta.

$\mathrm{Z}=-3.904-3.904 \leq 0.05$ por lo tanto la hipótesis nula se rechaza.

La Hipotesis de investigación o alterna se acepta.

\section{Conclusiones}

De las hipótesis de investigación planteada en este proyecto, se obtienen las siguientes conclusiones:

Hipótesis alterna: El uso de un Ambiente Virtual de Aprendizaje Moodle con objetos de aprendizaje o evaluación de un curso, mejora el nivel de desempeño de competencias del estudiante

Hipótesis nula: Los estudiantes que son evaluados con estrategias de aprendizaje presencial no alcanzan un mejor nivel de desempeño 
Al aplicar la técnica estadística U de Mann-Whitney a variables cualitativas ordinales como lo es el nivel de desempeño del estudiante (Excelente, Notable, Bueno, Suficiente e Insuficiente) y la variable cualitativa modalidad de aprendizaje (1: Presencial, 2: blearning) se obtiene el estadístico $\mathrm{z}$ menor al 0.05 por lo que la hipótesis nula se rechaza y la hipótesis de investigación o alterna es aceptada. También se muestra una descripción de los dos grupos comprados (presencial y blearning), la suma de rangos y un valor que representa cual grupo tiene una mediana mayor, este dato es el rango promedio, que se obtiene de dividir la suma de rangos de cada grupo entre la cantidad de casos en el grupo.

El uso de un Ambiente Virtual de Aprendizaje como herramienta de las clases presenciales convierte la actividad docente en una modalidad b-learning; queda demostrado que incluir objetos de aprendizaje bien diseñados como estrategias de aprendizaje en el Moodle, mejora el nivel de desempeño académico del estudiante de Ingeniería en Sistemas Computacionales de la asignatura de Simulación del Tecnológico de Ciudad Valles.

\section{Referencias}

Gamino-Carranza, A., \& Acosta-González, M. G. (2016). Modelo curricular del Tecnológico Nacional de México. Revista electrónica educare, 20(1), 212-236.

García-Ruiz, R., Aguaded, I., \& BartoloméPina, A. (2018). La revolución del blended learning en la educación a distancia. RIED. Revista Iberoamericana de Educación a Distancia, 21(1), 25-32.

Gutiérrez, M. A. U. (2017). Un modelo educativo crítico con enfoque de competencias. Documentos de trabajo, 3.

TecNM, (2015). Lineamiento para la Evaluación y Acreditación de Asignaturas Versión 1.0.

TecNM, (2012). Modelo Educativo para el Siglo XXI: Formación y Desarrollo de Competencias Profesionales
GARCIA-CHI, Rosa Imelda, HERNANDEZ, María Antonieta, IZAGUIRRE-CÁRDENAS, Nelly Rosina y EGUIA-ALVAREZ, Arturo. Uso de un ambiente virtual de aprendizaje en la ingeniería en sistemas herramienta de 